\title{
$n-3$ and $n-6$ Polyunsaturated fatty acids suppress sterol regulatory element binding protein activity and increase flow of non-esterified cholesterol in HepG2 cells
}

\author{
Mattia Di Nunzio ${ }^{1}$, Diederik van Deursen ${ }^{2}$, Adrie J. M. Verhoeven ${ }^{2}$ and Alessandra Bordoni ${ }^{1 *}$ \\ ${ }^{1}$ Department of Biochemistry 'G. Moruzzi', Research Centre on Nutrition and Vitamins, University of Bologna, Via Irnerio 48, \\ 40126 Bologna, Italy \\ ${ }^{2}$ Department of Biochemistry, Cardiovascular Research School COEUR, Erasmus MC, PO Box 2040, NL-3000 CA Rotterdam, \\ The Netherlands
}

(Received 18 March 2009 - Revised 16 July 2009 - Accepted 22 July 2009 - First published online 14 October 2009)

The plasma lipid-lowering effect of PUFA, one of their main beneficial effects, is considered to be related to the regulation of lipid biosynthesis through transcription factors including sterol regulatory element binding proteins (SREBP). In the present study, we compared the effect of different PUFA on SREBP activity in HepG2 cells, using a sterol regulatory element-luciferase reporter construct as a probe. Supplementation with different fatty acids reduced SREBP activity in the order $20: 5 n-3=18: 2 n-6=20: 4 n-6 \gg 18: 3 n-3=22: 6 n-3=22: 5 n-6 \gg 18: 1 n-9$. The suppression of SREBP activity greatly depended on the degree of incorporation of the supplemented PUFA into cellular lipids, and correlated positively with the unsaturation index $(r 0.831 ; P<0.01)$ of total cell lipids. Supplemented PUFA were also metabolised to longer and more unsaturated species. These processing activities were higher for $n-3$ than $n-6$ PUFA $(P<0 \cdot 01)$. We studied the effect of PUFA on the intracellular distribution of non-esterified cholesterol, using filipin staining and fluorescence microscopy with or without the cholesterol traffic blocker U18666A. The data show that the incorporation of PUFA increases non-esterified cholesterol flow from the plasma membrane to intracellular membranes. We conclude that suppression of SREBP activity by PUFA depends on the degree of incorporation into cellular lipids, and is associated with increased flow of non-esterified cholesterol between the plasma membrane and intracellular membranes.

PUFA: Sterol regulatory element binding proteins: Non-esterified cholesterol: Sterol regulatory elements: HepG2 cells

It is well documented that dietary intake of PUFA, and particularly a correct $n-6: n-3$ ratio, contributes to the prevention of many chronic diseases ${ }^{(1)}$. In order to explain how PUFA can influence so many biological processes, different mechanisms of action have been hypothesised, i.e. the modification of membrane fluidity and functionality through changes in membrane lipid composition ${ }^{(2)}$, the alteration of eicosanoid signalling and the modulation of gene expression ${ }^{(3-5)}$. Their main beneficial effect, i.e. plasma lipid lowering, is nowadays considered to be related to the regulation of lipid biosynthesis through the transcription factors $\mathrm{NF}-\mathrm{\kappa B}^{(6)}$, retinoid $\mathrm{X}$ receptor $^{(7)}, \operatorname{PPAR}^{(8)}$ and sterol regulatory element binding proteins (SREBP) ${ }^{(9)}$.

PUFA are thought to interact indirectly with SREBP ${ }^{(10,11)}$, but the mechanism of this interaction is still unclear. It is unlikely that PUFA affect SREBP through liver X receptor (LXR), a major activator of SREBP-1c transcription, as fish oil-fed rats showed suppression of hepatic SREBP-1c target genes, but not of LXR target genes such as cytochrome
P450 family 7 subfamily A polypeptide 1 (CYP7A1), ATPbinding cassette (ABC)-G5 or ABC-G8 transporters ${ }^{(12)}$. In addition, in hepatocytes the treatment with EPA $(20: 5 n-3)$ inhibited SREBP-1c-controlled genes both in the absence and the presence of a synthetic LXR agonist ${ }^{(13)}$. Alternatively, PUFA may act by decreasing SREBP mRNA stability ${ }^{(14,15)}$. A recent study showed that DHA (22:6n-3) reduces the abundance of the nuclear form of SREBP-1 (nSREBP-1) in rat hepatocytes through 26S-proteasome- and Erk-dependent pathways ${ }^{(16)}$.

Another possibility is that the SREBP suppression is linked to the incorporation of PUFA into cell membranes. Cholesterol is abundant in mammalian plasma membranes (PM), accounting for as much as $50 \%(\mathrm{~mol} / \mathrm{mol})$ of total lipid. It globally modulates the molecular organisation of the membrane, and its distinct affinity for different lipids drives the formation of membrane subdomains ${ }^{(17)}$. When PUFA are incorporated to a greater extent into membrane phospholipids, their poor affinity for cholesterol drives the formation of

Abbreviations: ALA, $\alpha$-linolenic acid; ARA, arachidonic acid; BSA, bovine serum albumin; LA, linoleic acid; NEC, non-esterified cholesterol; nSREBP, nuclear form of sterol regulatory element binding protein; OA, oleic acid; PM, plasma membrane; SREBP, sterol regulatory element binding protein; SRE-luc, sterol regulatory element-luciferase.

* Corresponding author: Dr Alessandra Bordoni, fax +39 051 2091235, email alessandra.bordoni@unibo.it 
PUFA-enriched and cholesterol-depleted subdomains ${ }^{(18)}$. Since maturation of SREBP is regulated by the non-esterified cholesterol (NEC) content of the endoplasmic reticulum membranes ${ }^{(19)}$, it is conceivable that PUFA inhibit SREBP processing by causing redistribution of NEC from the PM to the endoplasmic reticulum.

To test this hypothesis, we supplemented HepG2 cells with different concentrations of various PUFA, and we compared the degree of their incorporation into cell lipids with the activity of SREBP, measured by using a sterol regulatory element-luciferase (SRE-luc) construct as a probe. Furthermore, the distribution of NEC over the PM and intracellular membranes was assessed by fluorescence microscopy. We chose HepG2 cells as the model system since the liver plays a central role in the regulation of cholesterol homeostasis.

\section{Methods and materials}

\section{Materials}

Dulbecco's modified Eagle's medium and Dulbecco's PBS were purchased from Lonza (Breda, The Netherlands), and Reporter Gene assay lysis buffer from Roche (Almere, The Netherlands). Fetal calf serum and Lipofectamine Plus were from Invitrogen (Groningen, The Netherlands), and U18666A and fatty acids from Sigma (St Louis, MO, USA). All other chemicals and solvents were of the highest analytical grade. The SRE-luc reporter construct was generated by insertion of part of the hamster hydroxy-3-methylglutaryl-CoA synthase promoter region into pGL3-Basic ${ }^{(20)}$. pGAP-RL (a kind gift from Dr A. A. F. de Vries, Leiden, The Netherlands) contained part of the human glyceraldehyde 3-phosphate dehydrogenase (GAP) promoter, and was generated by insertion of the $0.5 \mathrm{~kb}$ HindIII-XhoI fragment of pGAP489CAT $^{(21)}$ into pRL-null.

\section{Methods}

HepG2 cells tissue culture. HepG2 human hepatoma cells were maintained in Dulbecco's modified Eagle's medium supplemented with $10 \%(\mathrm{v} / \mathrm{v})$ fetal calf serum, penicillin $(100 \mathrm{IU} / \mathrm{ml})$ and streptomycin $(100 \mu \mathrm{g} / \mathrm{ml})$ at $37^{\circ} \mathrm{C}, 95 \%$ air, $5 \% \mathrm{CO}_{2}$. Once per week, cells were split 1:10 into a new $75 \mathrm{~cm}^{2}$ flask; medium was refreshed once per week.

Fatty acid supplementation. Fatty acids were dissolved at different concentrations $(6,60$ and $120 \mu \mathrm{M}, \mathrm{w} / \mathrm{v})$ in $100 \%$ isopropanol, and bound to bovine serum albumin (BSA). Fatty acid-BSA complexes were prepared fresh each time at a final BSA concentration of $0.5 \%$ (in serum-free Dulbecco's modified Eagle's medium), and cells were incubated for $21 \mathrm{~h}$ with fatty acids. Final isopropanol concentration in the media was kept below $1 \%(\mathrm{v} / \mathrm{v})$. Control cells received corresponding amounts of BSA and isopropanol.

Determination of HepG2 fatty acid composition. Cells were seeded in six-well plates. After $24 \mathrm{~h}$, at $75-80 \%$ confluence, cells were supplemented with fatty acid-BSA complexes at $60 \mu \mathrm{M}$-fatty acid concentration. After $21 \mathrm{~h}$, cells were washed four times with ice-cold Dulbecco's PBS, scraped off and collected by centrifugation for $3 \mathrm{~min}$ at $1000 \mathrm{~g}$ and $4^{\circ} \mathrm{C}$. Total cellular lipids were extracted according to Folch et al. ${ }^{(22)}$, and methyl esterified according to Stoffel et al. ${ }^{(23)}$. The fatty acid composition (as methyl esters) was determined by GC (GC 8000; Fisons, Milan, Italy) using a capillary column (SP 2340; $0.2 \mu \mathrm{m}$ film thickness) at a programmed temperature gradient $\left(160-210^{\circ} \mathrm{C} ; 8^{\circ} \mathrm{C} / \mathrm{min}\right)$ as previously reported ${ }^{(24)}$.

Luciferase assay. Cells were seeded in twenty-four-well plates. After $24 \mathrm{~h}$, at $75-80 \%$ confluence, cells were transfected with SRE-luc (0.4 $\mu \mathrm{g}$ per well) and pGAP-RL (60 ng per well) using Lipofectamine Plus as described previously ${ }^{(20)}$. After $3 \mathrm{~h}$, the medium was refreshed and supplemented with fatty acids at 6,60 and $120 \mu \mathrm{M}(\mathrm{w} / \mathrm{v})$ concentrations. After $21 \mathrm{~h}$, cells were washed three times with ice-cold Dulbecco's PBS and lysed. Luciferase activity was determined in the cell extracts with the Dual-Glo luciferase assay kit (Promega, Leiden, The Netherlands) using a Packard Top Count NXT luminometer (Packard Instruments Co., Inc., Meriden, CT, USA). Data were normalised for Renilla activity measured in the same sample to account for differences in cell viability and transfection efficiency. At $120 \mu \mathrm{M}-\mathrm{PUFA}$, Renilla expression levels were similar for the different fatty acids used, and amounted to 5721 (SD 471) counts $(n$ 4) v. 6115 (SD 1986) counts $(n$ 4) without fatty acid supplementation $(P=0.713$; paired $t$ test $)$.

Filipin staining. HepG2 cells were seeded on coverslips. After $24 \mathrm{~h}$, cells at about $20 \%$ confluence were supplemented with $60 \mu \mathrm{M}$-fatty acid-BSA complex. In some experiments U18666A $(2 \mu \mathrm{g} / \mathrm{ml})$, a blocker of intracellular cholesterol trafficking $^{(25)}$, was also added. Cells were washed after $21 \mathrm{~h}$ incubation, and fixated using $3 \%$ paraformaldehyde in Dulbecco's PBS for $60 \mathrm{~min}$. Thereafter, the cells were washed three times, treated with $50 \mathrm{~mm}$-glycine in Dulbecco's PBS for $30 \mathrm{~min}$ to quench paraformaldehyde, and stained with filipin $(40 \mu \mathrm{g} / \mathrm{ml})$ for a further $30 \mathrm{~min}$. Coverslips were mounted on microscope slides and the epifluorescence was examined using an inverted Olympus IX50 microscope. Images were acquired and analysed using AnalySiS imaging software (Soft Imaging Systems, Münster, Germany) as previously described ${ }^{(26)}$.

\section{Statistical analysis}

Data are presented as mean values and standard deviations of at least three independent experiments. Differences were tested for statistical significance by unpaired Student's $t$ tests $(P<0 \cdot 05)$.

\section{Results}

The incorporation of supplemented PUFA into cell lipids was determined by GC (Table 1). All PUFA were readily incorporated at the expense of MUFA, while the molar content of SFA was not significantly affected. When incubated with linoleic acid (LA; $18: 2 n-6)$, $\alpha$-linolenic acid (ALA; $18: 3 n-3$ ), arachidonic acid (ARA; 20:4n-6) or EPA, the corresponding elongated and more unsaturated fatty acids accumulated as well, suggesting that these PUFA are further metabolised upon cellular uptake. Metabolic conversion of the $n-3$ fatty acids was more efficient than the $n-6$ fatty acids. In fact in LA-supplemented cells the products:precursor ratio was 0.29 (SD 0.04), compared with 0.70 (SD 0.13) in ALAsupplemented cells $(P<0 \cdot 01)$. Supplementation with oleic 
Table 1. Fatty acid composition (mol \%) of HepG2 cells in the control condition and after PUFA supplementation (Mean values and standard deviations for three independent experiments)

\begin{tabular}{|c|c|c|c|c|c|c|c|c|c|c|c|c|c|c|c|c|}
\hline \multirow[b]{4}{*}{ Cellular lipids } & \multicolumn{16}{|c|}{ Fatty acid supplemented } \\
\hline & & & \multirow{2}{*}{\multicolumn{2}{|c|}{$\frac{n-9}{18: 1(\mathrm{OA})}$}} & \multicolumn{6}{|c|}{$n-6$} & \multicolumn{6}{|c|}{$n-3$} \\
\hline & \multicolumn{2}{|c|}{ None } & & & \multicolumn{2}{|c|}{$18: 2(\mathrm{LA})$} & \multicolumn{2}{|c|}{$20: 4$ (ARA) } & \multicolumn{2}{|c|}{$22: 5$ (DPA) } & \multicolumn{2}{|c|}{$18: 3(A L A)$} & \multicolumn{2}{|c|}{$20: 5$ (EPA) } & \multicolumn{2}{|c|}{$22: 6(\mathrm{DHA})$} \\
\hline & Mean & SD & Mean & SD & Mean & SD & Mean & SD & Mean & SD & Mean & SD & Mean & SD & Mean & SD \\
\hline $14: 0$ & $4 \cdot 32$ & 0.56 & 3.86 & 1.02 & $4 \cdot 23$ & 0.5 & 4.74 & 0.93 & 4.42 & 0.39 & $4 \cdot 27$ & 0.57 & $4 \cdot 32$ & 0.21 & 3.94 & 0.65 \\
\hline $16: 0$ & 31.34 & 0.96 & 28.43 & 2.76 & 30.15 & 1.94 & 3.14 & 1.32 & 3.63 & 0.68 & 31.26 & 2.79 & 32.26 & 0.91 & 30.99 & 1.41 \\
\hline $16: 1 n-7$ & $10 \cdot 26$ & 0.32 & $8.44^{\star *}$ & 0.35 & $7.89^{\star \star}$ & 0.77 & $8 \cdot 28^{\star *}$ & 0.32 & 9.77 & 0.99 & $9 \cdot 17$ & 0.82 & 9.29 & 0.72 & 9.99 & 1.52 \\
\hline $18: 0$ & 8.37 & 0.73 & 8.74 & 2.01 & 9.09 & 2.58 & 8.04 & 0.5 & 8.88 & 2.44 & 9 & 1.94 & 7.69 & $1 \cdot 12$ & 9.37 & 2.87 \\
\hline $18: 1 n-7 / n-9$ & $40 \cdot 36$ & 0.39 & 44.89 & $2 \cdot 90$ & $32 \cdot 34^{\star \star}$ & 2.58 & $32 \cdot 44^{\star \star \star}$ & 0.69 & $37 \cdot 66^{\star}$ & 1.11 & $31.52^{\star \star}$ & 3.09 & $33.96^{\star \star \star}$ & 0.91 & $38 \cdot 02^{*}$ & 1.20 \\
\hline $18: 2 n-6$ & 1.24 & 0.12 & 1.34 & 0.21 & $10 \cdot 93^{\star \star \star}$ & $1 \cdot 16$ & 1.09 & 0.03 & $1 \cdot 30$ & 0.16 & 1.32 & 0.23 & 1.25 & 0.08 & 1.33 & 0.02 \\
\hline $18: 3 n-3$ & $1 \cdot 2$ & 0.15 & 1.50 & 0.19 & 0.98 & 0.18 & 0.94 & 0.13 & 1.12 & 0.13 & $7 \cdot 13^{\star \star}$ & 1.63 & 0.97 & 0.23 & $1 \cdot 16$ & 0.16 \\
\hline $20: 4 n-6$ & 1.6 & 0.19 & 1.62 & 0.5 & $3 \cdot 19^{\star \star}$ & 0.27 & $10.57^{\star \star}$ & 1.38 & 1.69 & 0.22 & 1.4 & 0.41 & 1.58 & 0.36 & 1.6 & 0.34 \\
\hline $20: 5 n-3$ & 0.14 & 0.03 & 0.13 & 0.07 & $0.05^{\star \star}$ & 0.01 & $0.04^{\star}$ & 0.03 & 0.11 & 0.02 & $2 \cdot 96^{* \star *}$ & 0.25 & $4.79^{\star \star \star}$ & 0.37 & 0.19 & 0.07 \\
\hline $22: 5 n-6$ & 0 & 0 & 0 & 0 & 0 & 0 & $0.71^{\star \star \star}$ & 0.34 & $2 \cdot 37^{\star \star \star}$ & 0.22 & 0 & 0 & 0 & 0 & 0 & 0 \\
\hline $22: 5 n-3$ & 0.16 & 0.03 & 0.18 & 0.08 & 0.18 & 0.05 & 0.20 & 0.05 & 0.13 & 0.02 & $0.75^{\star}$ & 0.25 & $1.95^{\star \star \star}$ & 0.23 & 0.16 & 0.02 \\
\hline $22: 6 n-3$ & 0.99 & 0.17 & 1.06 & 0.41 & 0.97 & 0.22 & 0.82 & 0.24 & 0.94 & 0.06 & 1.22 & 0.35 & $1.93^{\star \star}$ & 0.17 & $3 \cdot 26^{\star \star}$ & 0.46 \\
\hline
\end{tabular}

OA, oleic acid; LA, linoleic acid; ARA, arachidonic acid; DPA, docosapentaenoic acid; ALA, $\alpha$-linolenic acid. Mean value was significantly different from that of the control cells: ${ }^{\star} P<0.05,{ }^{* \star} P<0.01,{ }^{\star \star *} P<0.001$.

†Cells were incubated for $21 \mathrm{~h}$ with $60 \mu \mathrm{m}$-fatty acids. 

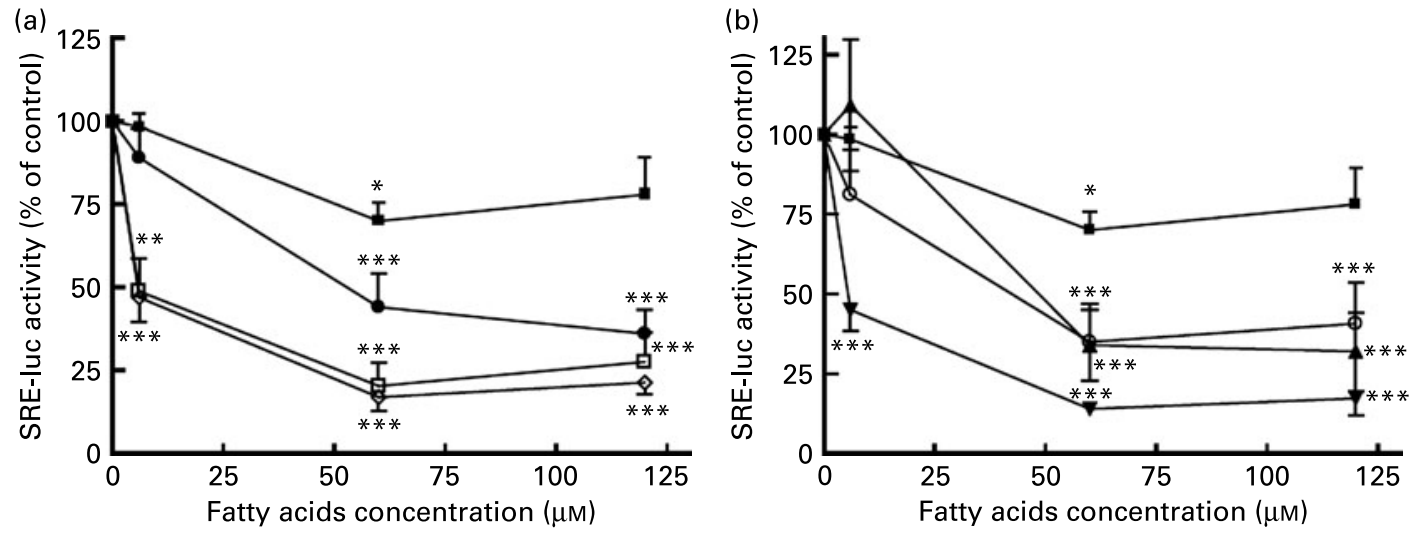

Fig. 1. Effect of $n-6$ and $n-3$ PUFA supplementation on sterol regulatory element-luciferase (SRE-luc) activity. HepG2 cells were incubated for $21 \mathrm{~h}$ with different concentrations of $n-6$ (a) and $n-3$ (b) fatty acids, and the effect on SRE-luc activity was determined. Fatty acids used were linoleic acid ( $\square$ ), arachidonic acid $(\diamond)$, docosapentaenoic acid $(\bullet), \alpha$-linolenic acid $(O)$, EPA $(\mathbf{\nabla})$ and DHA $(\mathbf{\Lambda})$. The effects of PUFA were compared with similar concentrations of oleic acid $(\boldsymbol{\square})$. Data are expressed as percentage of control from three separate experiments each performed in quadruplicate. Values are means, with standard deviations represented by vertical bars. Mean value was significantly different from that of the control cells: ${ }^{*} P<0.05,{ }^{* *} P<0.01,{ }^{* \star *} P<0.001$.

acid (OA; $18: 1 n-9)$ did not result in significant changes in the relative content of $\mathrm{OA}$, nor of any of the other fatty acids.

The effect of $n-6$ and $n-3$ PUFA on SRE-luc activity in HepG2 cells is shown in Fig. 1(a) and (b), respectively. PUFA were supplemented at a very low concentration $(6 \mu \mathrm{M})$ and at two physiological plasma concentrations $(60$ and $120 \mu \mathrm{M})$. Data were compared with the effects of corresponding concentrations of OA. OA did not cause any reduction of SRE-luc activity at $6 \mu \mathrm{M}$, and only a mild reduction at $60 \mu \mathrm{M}$. At $6 \mu \mathrm{M}$, LA and ARA reduced SRE-luc activity by $50 \%$ (Fig. 1(a)). Maximal inhibition of $75 \%$ was observed at 60 and $120 \mu \mathrm{M}$. Docosapentaenoic acid (22:5n-6) had no effect at the low concentration, while its inhibitory effect was similar to LA and ARA at $120 \mu \mathrm{M}$. Among $n$-3 PUFA (Fig. 1(b)), EPA appeared as effective as LA and ARA at all concentrations used. In contrast, the inhibitory effect of ALA and DHA was apparent only at 60 and $120 \mu \mathrm{M}$.

We then compared the degree of cellular lipid modification with the inhibition of SRE-luc activity obtained in $60 \mu \mathrm{M}$-PUFA-supplemented cells. Interestingly, the more a supplemented fatty acid or its metabolites were incorporated into total cellular lipids, the stronger was its effect on SREluc activity. An inverse correlation was present between SRE-luc activity and the total PUFA content of cellular membranes $(r-0.79 ; P=0.02)$ (Fig. 2(a)), and between SRE-luc activity and the unsaturation index of total cellular lipids $(r-0.83 ; P<0.01)$ (Fig. 2(b)). It is therefore evident that the enrichment of PUFA in HepG2 cells is correlated with the degree of suppression of SREBP activity.

To evaluate the intracellular distribution of NEC in HepG2 cells, filipin staining followed by fluorescence microscopy was performed in control and $60 \mu \mathrm{M}$-fatty acid-BSAsupplemented cells. In control cells, NEC appeared to be mainly localised at the cell periphery, in proximity to the PM, with a low intracellular staining intensity (Fig. 3(a)). The relative distribution of cholesterol was not affected by any of the supplemented PUFA, as illustrated for EPA in Fig. 3(b). Cholesterol is continuously transported from the PM to intracellular membranes, and vice versa. To estimate the effect of PUFA on the flow of cholesterol from the PM to intracellular membranes, we used U18666A, an inhibitor of cholesterol export from endo- and lysosomes ${ }^{(25)}$. In the presence of U18666A, filipin staining at the cell periphery was
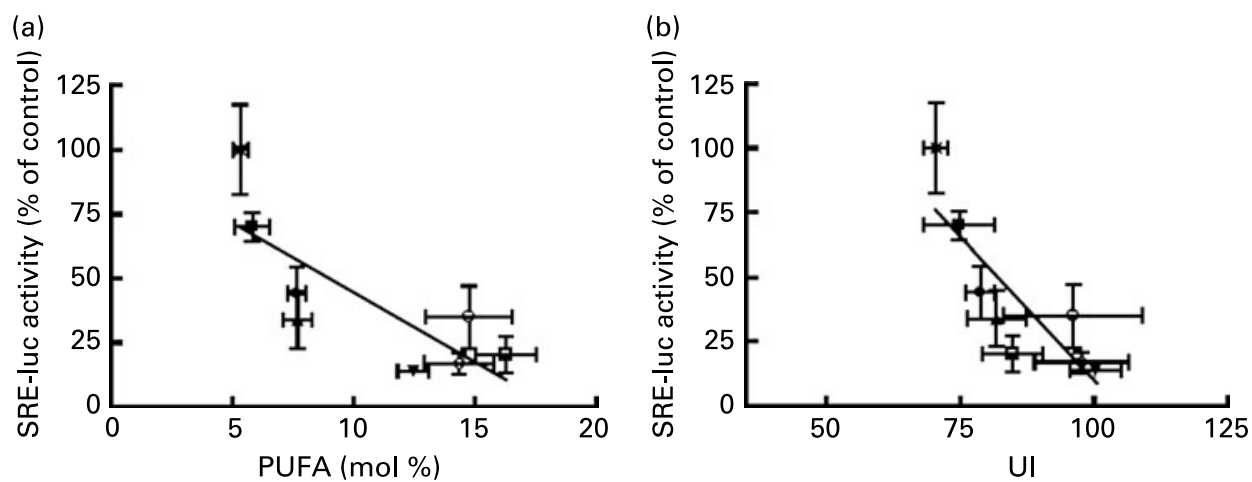

Fig. 2. Correlations (-) between sterol regulatory element-luciferase (SRE-luc) activity and cell membrane fatty acid composition. Cells were incubated for $21 \mathrm{~h}$ without $\left({ }^{\star}\right)$ or with $60 \mu \mathrm{M}$-fatty acids. Fatty acids used were oleic $(\boldsymbol{\square})$, linoleic $(\square)$, arachidonic $(\diamond)$, docosapentaenoic $(\bullet)$, $\alpha$-linolenic $(\bigcirc)$, eicosapentaenoic $(\nabla)$ and docosahexaenoic $(\boldsymbol{\Lambda})$ acids. Total lipid fatty acid composition was obtained by GC, as reported in Table 1. Unsaturation index (UI) was obtained by multiplying the relative molar content of each fatty acid by its number of double bonds, then adding up all obtained values. Values on PUFA content (a) or UI (b) and SRE-luc activity are means, with standard deviations represented by horizontal and vertical bars, respectively. SRE-luc activity negatively correlated with PUFA content $(r-0.79 ; P=0.02)$ and UI $(r-0.83 ; P<0.01)$. 


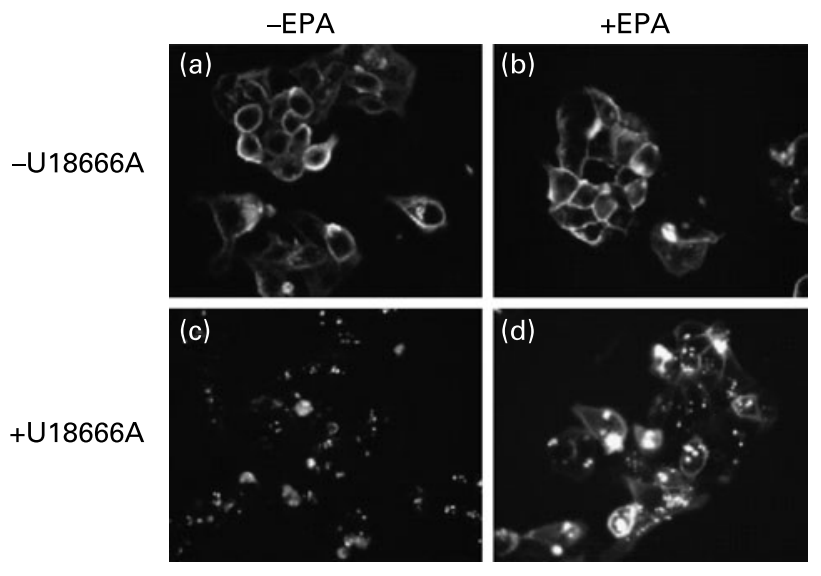

Fig. 3. Effect of EPA and U18666A on non-esterified cholesterol distribution in HepG2 cells. Cells were incubated for $21 \mathrm{~h}$ without $(\mathrm{a}, \mathrm{c})$ or with $60 \mu \mathrm{M}$ bovine serum albumin-bound EPA (b, d), and without $(a, b)$ or with U18666A $(2 \mu \mathrm{g} / \mathrm{ml})(\mathrm{c}, \mathrm{d})$, and then stained for non-esterified cholesterol by filipin. Data are representative of three independent experiments.

markedly reduced (Fig. 3(c)). Simultaneously, intracellular staining appeared as small clusters of bright spots, indicating that NEC is confined to intracellular organelles. The NEC that accumulated in the intracellular organelles apparently reflects the amount of NEC that has been transported from the PM during incubation with U18666A. When cells were co-incubated with U18666A and EPA (Fig. 3(d)), intracellular staining increased further and the cell periphery also stained strongly (compare Fig. 3(c) and (d)). Compared with U18666A alone, the number and size of the intracellular staining spots were markedly increased. The increased intracellular accumulation of NEC in EPA-supplemented cells indicates increased flow of cholesterol from the PM to endo- and lysosomes.

\section{Discussion}

It is 40 years since Keys \& Parlin ${ }^{(27)}$ developed predictive equations to quantify the effects of fatty acids and dietary cholesterol on plasma cholesterol concentrations, and dietary PUFA were reported as important regulators of cholesterol metabolism. It is widely accepted that PUFA modulate the expression of genes involved in lipid metabolism, and SREBP have emerged as key mediators of this regulation. Nevertheless, the exact mechanism(s) by which PUFA interact with SREBP is still unclear, as well as the effectiveness of different $n-6$ and $n-3$ PUFA. To clarify this, we supplemented HepG 2 cells with both $n-6$ and $n-3$ PUFA, while the monounsaturated OA was used for comparison.

The degree of incorporation of supplemented PUFA and their metabolites appeared to be an important determinant of their inhibitory effect on SREBP activity. In fact, the more they were incorporated into total cellular lipids, the lower was SREBP activity. This is further illustrated by the inverse correlation between SREBP activity and the unsaturation index of cellular lipids. It is therefore evident that suppression of SREBP activity by PUFA in HepG2 cells depends on the degree of their enrichment in cellular lipids. Supplementation with $60-120 \mu \mathrm{M}-\mathrm{PUFA}$ reduced SREBP activity in the order $\mathrm{EPA}=\mathrm{LA}=\mathrm{ARA} \gg \mathrm{ALA}=\mathrm{DHA}=$ docosapentaenoic acid.
In a recent review Jump et al. ${ }^{(28)}$ indicated DHA as the most active PUFA in modulating hepatic gene transcription and the most potent suppressor of SREBP-1 nuclear abundance. Actually, in rat primary hepatocytes, DHA was more effective than EPA but ineffective below $100 \mu \mathrm{M}$ in reducing nSREBP-1 protein ${ }^{(16)}$. Worgall et al. ${ }^{(29)}$ found that LA, ARA, ALA and docosapentaenoic acid were similarly effective in HepG2 cells at $300 \mu \mathrm{M}$; EPA and DHA were not included in this study. In rat hepatoma cells, ARA, EPA and DHA $(150 \mu \mathrm{M})$ were more effective in suppressing nSREBP-1 protein than LA and ALA $(300 \mu \mathrm{M})^{(30)}$. These differences in efficacy may be due to the different experimental models or the different PUFA:albumin ratios used. In addition, the reporter assay used in the present study does not discriminate between SREBP isoforms, and some PUFA may also suppress nSREBP-2.

Supplemented PUFA were not only incorporated into cell lipids as such, but they were also converted to longer and more unsaturated species by the HepG2 cells. This is in contrast to Yu-Poth et al. ${ }^{(31)}$ but in agreement with El-Badry et al. ${ }^{(32)}$. In accordance with this last paper, we found these processing activities to be higher for $n-3$ than $n$-6 PUFA, as indicated by the higher product:precursor ratios for $18: 3 n-3$ than $18: 2 n-6$.

Our fluorescence microscopy studies are consistent with an increased flow of NEC from the PM to intracellular membranes in PUFA-supplemented HepG2 cells. It is conceivable that incorporation of PUFA displaces cholesterol from the PM, thus increasing NEC flow to intracellular membranes. In the PM cholesterol is associated with sphingomyelin ${ }^{(29)}$. In HL-60 cells and human neutrophils, the increase in PUFA concentration stimulates neutral sphingomyelinase activity $^{(33,34)}$. Treatment of cultured cells with sphingomyelinase, thereby degrading the major raft sphingolipids, leads to a rapid increase in intracellular cholesterol content and subsequent inhibition of SREBP maturation ${ }^{(35)}$. In control cells, NEC appeared to be mainly localised at the cell periphery, in proximity to the PM, with a low intracellular localisation. The similar staining pattern observed in PUFA-supplemented cells argues against a major re-distribution of NEC from the PM to intracellular membranes. However, cholesterol in the PM is continuously turning over and it is estimated that the entire PM cholesterol pool cycles to the endoplasmic reticulum and back with a half-time of $40 \mathrm{~min}^{(36)}$. Therefore, suppression of SREBP activity may result from subtle increases in intracellular NEC not detectable by filipin staining. However, in the presence of U18666A, which inhibits NEC basal movements, the increased accumulation of NEC in intracellular organelles strongly suggests increased trafficking of cholesterol between the PM and intracellular compartments. Hence, it may be the NEC dynamics in the intracellular membranes rather than the NEC concentration that affects the post-translational maturation of SREBP.

Taken together, our data suggest that both $n-3$ and $n-6$ PUFA suppress SREBP activity in HepG2 cells via the increased flow of NEC from the PM to intracellular membranes, in proportion to their accumulation in cellular lipids. Besides, the regulation of SREBP activity by PUFA may also involve other mechanisms, such as sphingomyelin hydrolysis and consequent ceramide production ${ }^{(37)}$, PPAR activation $^{(38)}$, and accelerated degradation of nSREBP by 
a $26 \mathrm{~S}$ proteasome-dependent pathway $^{(16)}$, mechanisms that may or may not be secondary to PUFA incorporation into cellular membranes. Lowering of liver SREBP-1 protein levels by PUFA-enriched oil feeding has been consistently reported for rats and mice ${ }^{(16,39-40)}$, suggesting that our findings may hold also in vivo. Although additional studies are needed to elucidate how PUFA control SREBP activity, and thereby lipid metabolism, our data may contribute to the further development of PUFA as nutritional therapeutic agents for management of cholesterol and lipid homeostasis.

\section{Acknowledgements}

The authors would like to thank Dick H. W. Dekkers, Christina H. Lim and Ben C. Tilly for their helpful discussion, and Granarolo SpA (Bologna, Italy) for the financial support.

Part of the present study was supported by grants from the Programma Marco Polo, University of Bologna (M. di N.), the Dutch Heart Foundation (grant no. 2001B174) and Granarolo SpA (Bologna, Italy).

M. Di N. and D. v. D. performed the analysis. A. J. M. V. and A. B. designed and supervised the study, and wrote the paper.

All authors state that there is no conflict of interest associated with the present study.

\section{References}

1. Simopoulos AP (2008) The importance of the omega-6/omega-3 fatty acid ratio in cardiovascular disease and other chronic diseases. Exp Biol Med 233, 674-688.

2. Rajamoorthi K, Petrache HI, McIntosh TJ, et al. (2005) Packing and viscoelasticity of polyunsaturated $\omega-3$ and $\omega-6$ lipid bilayers as seen by ${ }^{2} \mathrm{H}$ NMR and X-ray diffraction. $J$ Am Chem Soc 127, $1576-1588$.

3. Worgall TS \& Deckelbaum RJ (1999) Fatty acids: links between genes involved in fatty acid and cholesterol metabolism. Curr Opin Clin Nutr Metab Care 2, 127-133.

4. Benatti P, Peluso G, Nicolai R, et al. (2004) Polyunsaturated fatty acids: biochemical, nutritional and epigenetic properties. J Am Coll Nutr 23, 281-302.

5. Sampath H \& Ntambi JM (2005) Polyunsaturated fatty acid regulation of genes of lipid metabolism. Апnи Rev Nutr 25, 317-340.

6. Zhao Y, Joshi-Barve S, Barve S, et al. (2004) Eicosapentaenoic acid prevents LPS-induced TNF- $\alpha$ expression by preventing NF-kB activation. $J$ Am Coll Nutr 23, 71-78.

7. Lengqvist J, Mata De Urquiza A, Bergman AC, et al. (2004) Polyunsaturated fatty acids including docosahexaenoic and arachidonic acid bind to the retinoid $\mathrm{X}$ receptor $\alpha$ ligandbinding domain. Mol Cell Proteomics 3, 692-703.

8. Edwards IJ \& O'Flaherty JT (2008) Omega-3 fatty acids and PPAR $\gamma$ in cancer. PPAR Res 2008, 358052.

9. König B, Spielmann J, Haase K, et al. (2008) Effects of fish oil and conjugated linoleic acids on expression of target genes of $\operatorname{PPAR} \alpha$ and sterol regulatory element-binding proteins in the liver of laying hens. Br J Nutr 100, 355-363.

10. Nakamura MT, Cheon Y, Li Y, et al. (2004) Mechanisms of regulation of gene expression by fatty acids. Lipids $\mathbf{3 9}$, 1077-1083.

11. Bordoni A, Di Nunzio M, Danesi F, et al. (2006) Polyunsaturated fatty acids: from diet to binding to PPARs and other nuclear receptors. Genes Nutr 1, 95-106.
12. Pawar A, Botolin D, Mangelsdorf DJ, et al. (2003) The role of liver $X$ receptor- $\alpha$ in the fatty acid regulation of hepatic gene expression. J Biol Chem 278, 40736-40743.

13. Nakatani T, Katsumata A, Miura S, et al. (2005) Effects of fish oil feeding and fasting on LXR $\alpha / R X R \alpha$ binding to LXRE in the SREBP-1c promoter in mouse liver. Biochim Biophys Acta 1736, 77-86.

14. Hannah VC, Ou J, Luong A, et al. (2001) Unsaturated fatty acids down-regulate SREBP isoforms $1 \mathrm{a}$ and $1 \mathrm{c}$ by two mechanisms in HEK-293 cells. $J$ Biol Chem 276, $4365-4372$.

15. Jump DB (2002) Dietary polyunsaturated fatty acids and regulation of gene transcription. Curr Opin Lipidol 13, 155-164.

16. Botolin D, Wang Y, Christian B, et al. (2006) Docosahexaneoic acid $(22: 6, n-3)$ regulates rat hepatocyte SREBP-1 nuclear abundance by Erk- and $26 \mathrm{~S}$ proteasome-dependent pathways. J Lipid Res 47, 181-192.

17. Silvius JR (2003) Role of cholesterol in lipid raft formation: lessons from lipid model systems. Biochim Biophys Acta 1610, 174-183.

18. Stillwell W, Shaikh SR, Zerouga M, et al. (2005) Docosahexaenoic acid affects cell signaling by altering lipid rafts. Reprod Nutr Dev 45, 559-579.

19. Weber LW, Boll M \& Stampfl A (2004) Maintaining cholesterol homeostasis: sterol regulatory element-binding proteins. World J Gastroenterol 10, 3081-3087.

20. Botma GJ, van Deursen D, Vieira D, et al. (2005) Sterolregulatory-element binding protein inhibits upstream stimulatory factor-stimulated hepatic lipase gene expression. Atherosclerosis 179, 61-67.

21. Aki T, Yanagisawa S \& Akanuma H (1997) Identification and characterization of positive regulatory elements in the human glyceraldehyde 3-phosphate dehydrogenase gene promoter. J Biochem 122, 271-278.

22. Folch J, Lees M \& Sloane Stanley GH (1957) A simple method for the isolation and purification of total lipides from animal tissues. J Biol Chem 226, 497-509.

23. Stoffel W, Chu F \& Ahrens EH Jr (1959) Analysis of long chain fatty acids by gas-liquid chromatography. Anal Chem 31, 307-308.

24. Bordoni A, Angeloni C, Leoncini E, et al. (2005) Hypoxia/reoxygenation alters essential fatty acids metabolism in cultured rat cardiomyocytes: protection by antioxidants. Nutr Metab Cardiovasc Dis 15, 166-173.

25. Härmälä AS, Pörn MI, Mattjus P, et al. (1994) Cholesterol transport from plasma membranes to intracellular membranes is inhibited by $3 \beta$-[2-(diethylamino)ethoxy]androst-5-en-17one. Biochim Biophys Acta 1211, 317-325.

26. Lim CH, Bijvelds MJ, Nigg A, et al. (2007) Cholesterol depletion and genistein as tools to promote F508delCFTR retention at the plasma membrane. Cell Physiol Biochem 20, 473-482.

27. Keys A \& Parlin RW (1966) Serum cholesterol response to changes in dietary lipids. Am J Clin Nutr 19, 175-181.

28. Jump DB, Botolin D, Wang Y, et al. (2008) Docosahexaenoic acid (DHA) and hepatic gene transcription. Chem Phys Lipids 153, 3-13.

29. Worgall TS, Sturley SL, Seo T, et al. (1998) Polyunsaturated fatty acids decrease expression of promoters with sterol regulatory elements by decreasing levels of mature sterol regulatory element-binding protein. $J$ Biol Chem 273, 25537-25540.

30. Le Jossic-Corcos C, Gonthier C, Zaghini I, et al. (2005) Hepatic farnesyl diphosphate synthase expression is suppressed by polyunsaturated fatty acids. Biochem J 385, 787-794.

31. Yu-Poth S, Yin D, Kris-Etherton PM, et al. (2005) Long-chain polyunsaturated fatty acids upregulate LDL receptor protein 
expression in fibroblasts and HepG2 cells. $J$ Nutr $\mathbf{1 3 5}$ 2541-2545.

32. El-Badry AM, Graf R \& Clavien PA (2007) Omega 3 - omega 6: What is right for the liver? $J$ Hepatol 47, 718-725.

33. Jayadev S, Linardic CM \& Hannun YA (1994) Identification of arachidonic acid as a mediator of sphingomyelin hydrolysis in response to tumor necrosis factor $\alpha$. J Biol Chem 269, 5757-5763.

34. Robinson BS, Hii CS, Poulos A, et al. (1997) Activation of neutral sphingomyelinase in human neutrophils by polyunsaturated fatty acids. Immunology 91, 274-280.

35. Simons K \& Ikonen E (2000) How cells handle cholesterol. Science 290, 1721-1726.

36. Liscum L \& Munn NJ (1999) Intracellular cholesterol transport. Biochim Biophys Acta 1438, 19-37.
37. Worgall TS, Johnson RA, Seo T, et al. (2002) Unsaturated fatty acid-mediated decreases in sterol regulatory element-mediated gene transcription are linked to cellular sphingolipid metabolism. J Biol Chem 277, 3878-3885.

38. König B, Koch A, Spielmann J, et al. (2007) Activation of PPAR $\alpha$ lowers synthesis and concentration of cholesterol by reduction of nuclear SREBP-2. Biochem Pharmacol 73, 574-585.

39. Sekiya M, Yahagi N, Matsuzaka T, et al. (2003) Polyunsaturated fatty acids ameliorate hepatic steatosis in obese mice by SREBP-1 suppression. Hepatology 38, 1529-1539.

40. Xu J, Cho H, O'Malley S, et al. (2002) Dietary polyunsaturated fats regulate rat liver sterol regulatory element binding proteins1 and -2 in three distinct stages and by different mechanisms. J Nutr 132, 3333-3339. 